\title{
CSRR-DGS Bandpass Filter Based on Half Mode Substrate Integrated Waveguide for X-Band Applications
}

\author{
Benzerga Fellah'1,3, Nabil Cherif2, Mehadji Abri ${ }^{3}$ and Hadjira Badaoui ${ }^{3}$ \\ ${ }^{1}$ Electrotechnical Department, Mustapha Stambouli University, Mascara, Algeria. \\ ${ }^{2}$ LSTE Laboratory, Mustapha Stambouli University, Mascara, Algeria \\ ${ }^{3}$ STIC Laboratory, Abou-Bekr Belkaid University, Tlemcen, Algeria. \\ Corresponding author: FELLAH Benzarga (e-mail: b.fellah@univ-mascara.dz)
}

ABSTRACT In this research, a novel half mode substrate integrated waveguide (HMSIW) bandpass filter based on defected ground structure cells (DGS) is proposed. By using the periodic square Complementary Split Ring Resonator (CSRR) resonant properties of DGS according to design requirement, an X-band bandpass filter is designed and analyzed to meet compact size, low insertion loss, and high rejection. The simulation results obtained by CST and HFSS simulators in $\mathrm{X}$-band show that the proposed filter is characterized by a large transmitted bandwidth of about $1.6 \mathrm{GHz}$ from 13.2 to 14.8 GHz. The higher simulated insertion loss is about $-2.6 \mathrm{~dB}$ and the lower return loss is about $-39 \mathrm{~dB}$. The proposed filter size is $9.5 \times 38.0 \mathrm{~mm}^{2}$ which make it a compact component. The filter design is optimized using CST and to validate the proposed filter the simulation results is compared by HFSS. The HFSS simulation results are in decent promise with the CST results.

INDEX TERMS Substrate integrated waveguide, band-pass filter, defected ground structure, CSRR.

\section{INTRODUCTION}

$S^{u}$ ubstrate integrated waveguide (SIW) was first proposed in 2003 [1]. Based on the TEn 0 transmission mode, SIW replaces the side metallic walls of the classic rectangular waveguide with two rows of metallic via holes, which converts conventional waveguides into planar structures [2-4]. Therefore, SIW not only have the properties of high quality factor and low radiation loss which are similar to metallic waveguides but also have the prominent advantage of compact size due to their planar physical structure. SIW have been applied to the design of filters [5]. Couplers [6], oscillators [7], power dividers [8], and many other microwave components.

Defected Ground Structure (DGS) is a geometrical periodic and/or aperiodic slots engraved on the ground plane of microwave circuits. The defects on the ground plane disturb the current distribution of the ground plane; this disturbance changes the characteristics of a structure by including some parameters (slot resistance, slot capacitance, and slot inductance) to the structure parameters [9].

The half mode substrate integrated waveguide (HMSIW) is used for reduces to SIW components size by the half or so and keeps the same performance of full SIW, it has been developed and widely used in varied applications. [10-12] Complementary split ring resonator (CSRR) was proposed in 2004 as a 3D metamaterial [13] that can exhibit negative permeability near its resonant frequency and therefore can be considered as a composite right/left-handed (CRLH) structure. When CSRRs are employed in SIW, pass bands based on the evanescent mode below the cutoff frequency of the SIW can be created $[14,16]$, which can further miniaturize the size of SIW microwave devices $[17,18]$. This paper describes a compact BPF for the TE10 mode based on periodic CSRR as DGS cells and by using HMSIW technique we reduced the filter size by half. We have obtained a good results for X-band in term of losses, size and rejection.

\section{SIW WAVEGUIDE DESIGN METHOD}

The main parameters in SIW design are the SIW width "W", the diameter "d" of the metal vias and the spacing "p" between the centers of two successive vias [19].

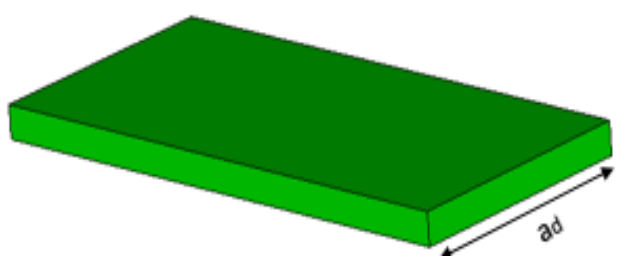

(a)

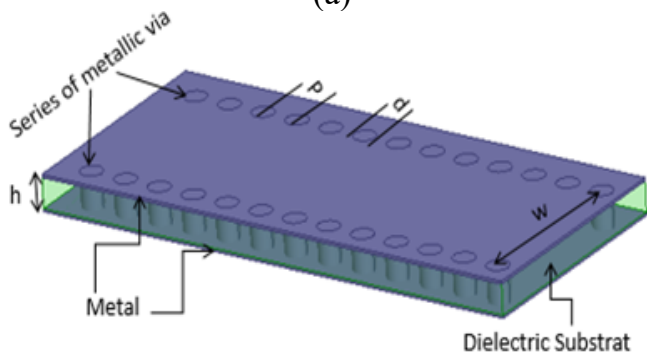

(b) 
FIGURE 1. (a) Dielectric Filled Waveguide and (b) SIW configurations.

The dielectric filled waveguide DFW is characterized by the dimension "ad" which is given by equation 1 .

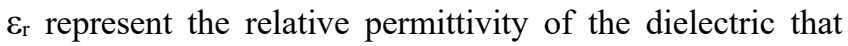
fills the waveguide [19].

$$
a_{d}=\frac{a}{\sqrt{\varepsilon_{r}}}
$$

For TE10 mode the cut-off frequency (fc) for a DFW is calculated by equation 2 [19].

$$
f_{c}=\frac{c}{2 a_{d}}
$$

Where $\mathrm{c}$ is the speed of light in a vacuum.

The optimal width of the SIW is calculated by equation 3 [20].

$$
W=a_{d}+\frac{d^{2}}{0.95 p}
$$

Two conditions are required for minimize the losses due to the field leakage from the gaps between vias:

$\mathrm{d}<\lambda \mathrm{g} \times 0.2$ and $\mathrm{p} \leq 2 \times \mathrm{d}$ where $\lambda \mathrm{g}$ is the guided wavelength [20].

$$
\lambda g=\frac{2 \pi}{\sqrt{\frac{(2 \pi f)^{2} \varepsilon_{r}}{c^{2}}-\left(\frac{\pi}{a}\right)^{2}}}
$$

\section{DGS SIW BAND-PASS FILTER DESIGN}

Using the CST we designed a new SIW-DGS band-pass filter structure for X-band on Arlon $\mathrm{Cu}$ Clad 217 based substrate having a loss tangent approximately 0.018 , a relative permittivity of 2.2 , a thickness $\mathrm{h}$ of $0.508 \mathrm{~mm}$ and the thickness of the conductor is $0.05 \mathrm{~mm}$ and for reduced the filter size we used filter half mode technique. The DGS CSRR inserted at the top plane are shown in the figure 2. We designed the filter based on two cascading CSRR in the top plane separated by a distance $\mathrm{M}$ of $5.5 \mathrm{~mm}$ and for impedance matching we used tapered transition.
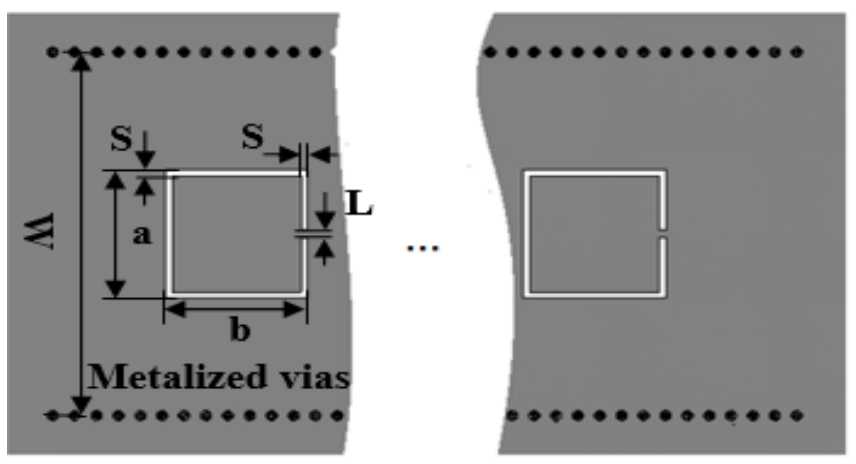

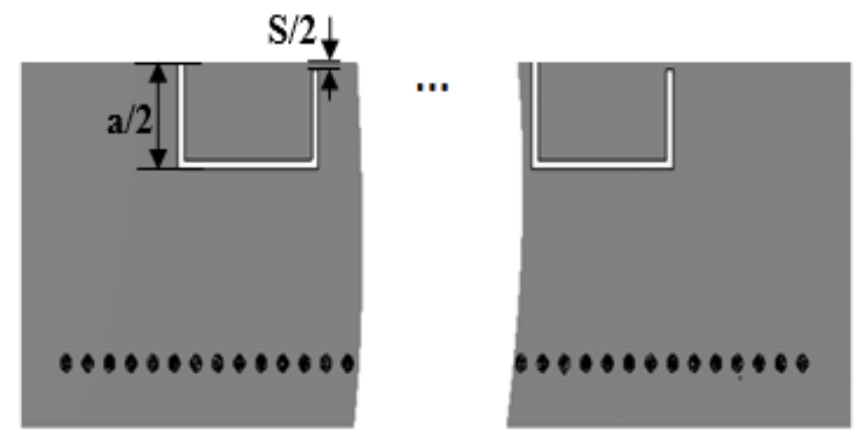

(b)

FIGURE 2. The proposed CSRR for (a) SIW and (b) HMSIW.

TABLE I. The optimal values of the proposed filter

\begin{tabular}{cccc}
\hline Parameters & Value $(\mathrm{mm})$ & Parameters & Value $(\mathrm{mm})$ \\
\hline $\mathrm{S}$ & 0.3 & $\mathrm{~b}$ & 6.5 \\
$\mathrm{~L}$ & 0.3 & $\mathrm{~W}$ & 18 \\
$\mathrm{a}$ & 6.5 & $\mathrm{~h}$ & 0.508 \\
$\mathrm{M}$ & 5.5 & $\mathrm{~d}$ & 0.8 \\
\hline
\end{tabular}

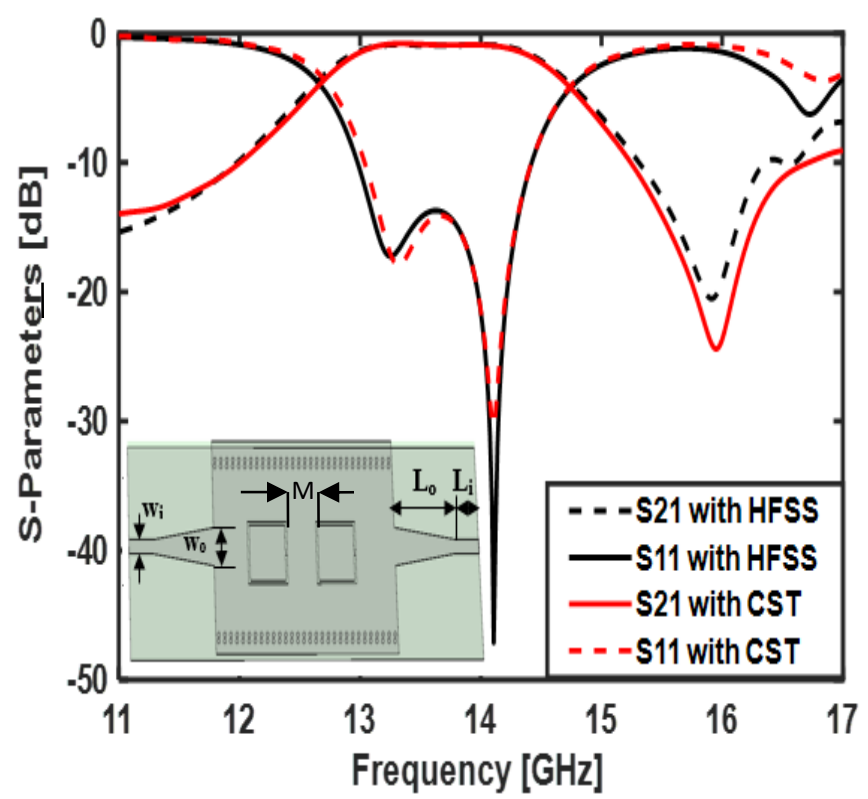

FIGURE 3. Transmission coefficient and return loss of the proposed filter with: $\mathrm{Wi}=1.55 \mathrm{~mm}, \mathrm{Wo}=3 \mathrm{~mm},=2.8 \mathrm{~mm}$ and $\mathrm{LO}=4 \mathrm{~mm}$.

From Figure 3 we observe that the insertion loss is about of $1.6 \mathrm{~dB}$ in band ranging from $13.1 \mathrm{GHz}$ to $14.3 \mathrm{GHz}$ also we observe the appearance of two peaks, the best reached $-47 \mathrm{~dB}$ at the frequency of $14.1 \mathrm{GHz}$. The rejection is fair under $13 \mathrm{GHz}$ and above 14.5. From this analysis it can be said that this SIW filter is functional in the $\mathrm{X}$ band with Fair performance. By using the half-mode technique, we reduced the filter size and kept the same performance of the SIW filter. The half mode SIW band pass filter structure and simulation are shown in the Figure 4.

(a) 


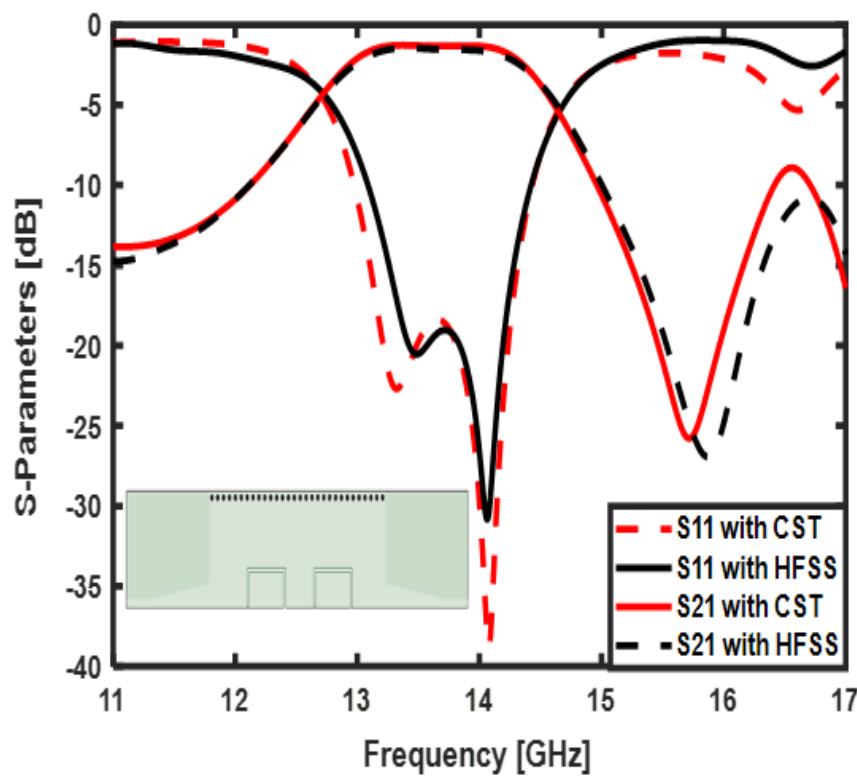

FIGURE 4. Transmission coefficient and return loss of HMSIW filter

From the previous figure we observe that: the transmission energy level is under $-7 \mathrm{~dB}$ in the band ranging from 11 to $12.7 \mathrm{GHz}$ and the reflection is maximum. In the band [13.1- $14.2 \mathrm{GHz}]$ the transmission is increased, and it reaches $-1.8 \mathrm{~dB}$ and the reflection decreased also several resonance frequency has been appeared the best reaches $-37 \mathrm{~dB}$ around the frequency of $14.1 \mathrm{GHz}$. and the rejection level is good above $14.3 \mathrm{GHz}$ and it reached $-27 \mathrm{~dB}$ at the frequency of $15.7 \mathrm{GHz}$.

In the next step we have cascaded three DGS cells in the top plane with a Separated distance of $4.86 \mathrm{~mm}$.

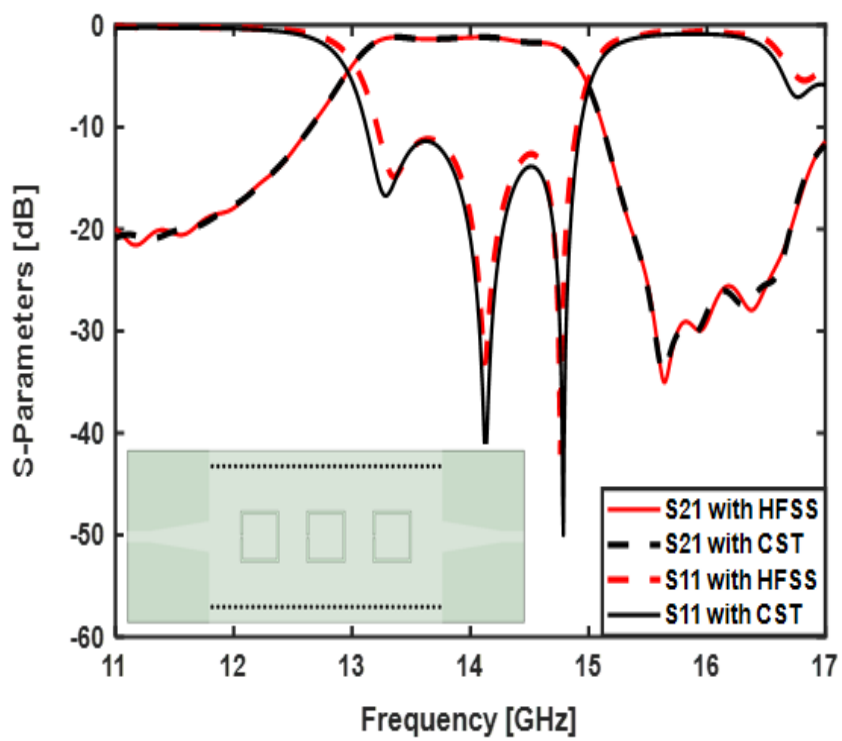

FIGURE 5. Transmission coefficient and return loss of DGS SIW filter

From the figure 5 it is show that good results are obtained. The simulation results show a good agreement of the cut-off frequency that is of $13 \mathrm{GHz}$. We observe a good agreement in the filtered band ranging from 13.1 to 15.1
$\mathrm{GHz}$ with an insertion loss of about $-1.5 \mathrm{~dB}$ in the transmitted bandwidth and the recorded return loss reached $-50 \mathrm{~dB}$ at the frequency of $14.8 \mathrm{GHz}$ and the rejection stretching from15.2 GHz until $17 \mathrm{GHz}$. By using the halfmode technique we reduced the filter size and kept the same performance of the SIW filter. The half mode SIW band pass filter structure and simulation are shown in the Figure 6.

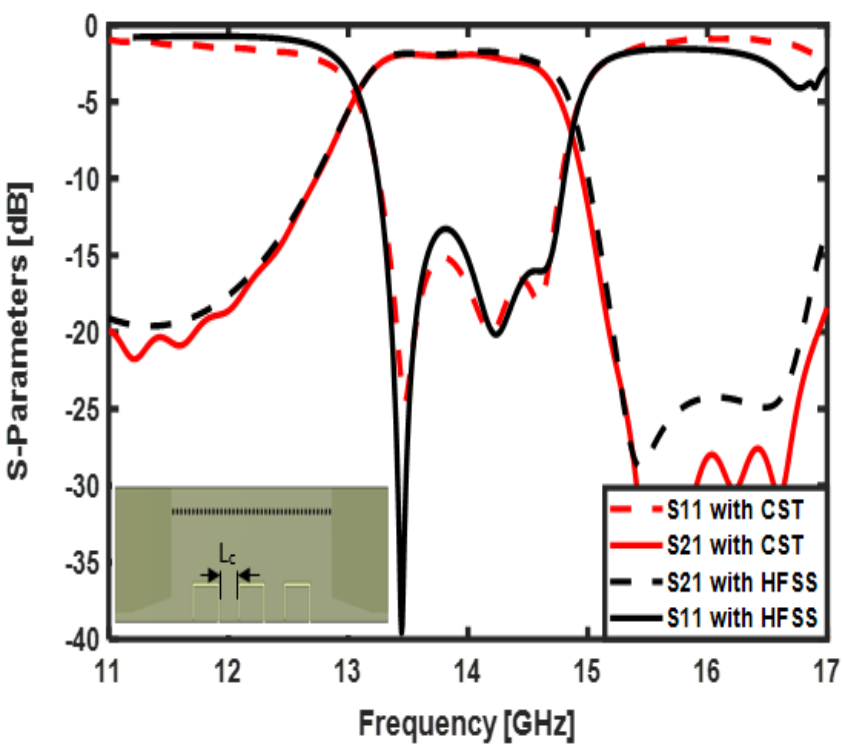

FIGURE 6. Transmission coefficient and return loss of HMSIW filter with: $\mathrm{LC}=4.86 \mathrm{~mm}$.

It can also be noted that we have an improvement in the rejection bands that ranging from 11 to $13.2 \mathrm{GHz}$ and from 14.8 to $17 \mathrm{GHz}$ also the return loss is under $-14 \mathrm{~dB}$ in all filtering band about insertion loss is about of $-2.6 \mathrm{~dB}$ in the transmitted band. We obtained a good agreement between CST Studio and Ansoft HFSS results which demonstrates our proposed filter.

\section{v. CONCLUSION}

In this work a band-pass filter based on HMSIW and periodic CSRR is designed and simulated for X-band applications, the design method is discussed. CST and HFSS simulators are used for the simulation, and we have compared between the results obtained. The proposed filter is characterized by a good performance in term of insertion loss, return loss, size and rejection. This type of filter is easy for integration with other planar circuit compared with conventional waveguide.

For future work we will work to reduce the size of the proposed filter and improve its losses and its rejection. 


\section{REFERENCES}

[1] D. Deslandes and K. Wu, "Single-substrate integration technique of planar circuits and waveguide filters," IEEE Transactions on Microwave Theory and Techniques, vol. 51, no. 2, pp. 593-596, 2003.

[2] X.-P. Chen and K. Wu, "Substrate integrated waveguide filters:design techniques and structure innovations," IEEE Microwave Magazine, vol. 15, no. 6, pp. 121-133, 2014.

[3] P. Li, H. Chu, and R. S. Chen, "SIW magic-T with bandpass response," Electronics Letters, vol. 51, no. 14, pp. 1078-1080, 2015.

[4] M. Esmaeili and J. Bornemann, "Substrate integrated waveguide dual- stopband filter," Microwave and Optical Technology Letters, vol. 56, no. 7, pp. 1561-1563, 2014.

[5] N. Cherif, M. Abri, F. Benzerga, H. Badaoui, and J. Tao, "A Compact Wideband DGS Bandpass Filter Based on Half Mode Substrate Integrated Waveguide Technology," International Journal of Microwave and Optical Technology, Vol.16, No.2, pp.142-147, (2021).

[6] B. Liu, W. Hong, Y.-Q. Wang, Q.-H. Lai, and K. Wu, " Half mode substrate integrated waveguide (HMSIW) 3-dB coupler," IEEE Microwave and Wireless Components Letters, vol. 17, no. 1, pp. 22-24, 2007.

[7] Zhe Chen, Wei Hong, Jixin Chen, Linsheng Li, " Design of A Push-Push and Push-Pull Oscillator Based on SIW/SICL Technique" IEEE Microwave and Wireless Components Letters, Vol.24, Iss. 6, pp.397 - 399, June 2014.

[8] B. Fellah, M. Abri and H. Badaoui," Optimized Bends, Corporate $1 \times 4$ and $1 \times 8$ SIW Power Dividers Junctions Analysis for V-Band Applications Using a Rigorous Finite Element Method, "Arabian Journal for Science and Engineering, Vol. 41, No. 9, pp. 3335-3343, Sept. 2015.

[9] M. K. Khandelwal , B. K. Kanaujia, and S. Kumar, " Defected Ground Structure: Fundamentals, Analysis, and Applications in Modern Wireless Trends" hindawi International Journal of Antennas and Propagation, Vol. 2017, pp.1-22, Feb 2017.

[10] W. Hong, B. Liu, Y. Wang et al., " Half mode substrate integrated waveguide: a new guided wave structure for microwave and millimeter wave application," in Proceedings of the 31st International Conference on Infrared and Millimeter Waves and 14th International Conference on Terahertz Electronics, p. 219, Shanghai, China, September 2006.
[11] B. Liu, W. Hong, Y.-Q. Wang, Q.-H. Lai, and K. Wu, "Half mode substrate integrated waveguide (HMSIW) 3-dB coupler,' IEEE Microwave and Wireless Components Letters, vol. 17, no. 1, pp. 22-24, 2007.

[12] Y. Wang, W. Hong, Y. Dong et al., "Half mode substrate integrated waveguide (HMSIW) bandpass filter," IEEE Microwave and Wireless Components Letters, vol. 17,no. 4, pp.265-267,2007.

[13] J. D. Baena, J. Bonache, F. Mart'in et al., " Equivalent-circuit models for split-ring resonators and complementary splitring resonators coupled to planar transmission lines," IEEE Transactions on Microwave Theory and Techniques, vol. 53, no. 4, pp. 1451-1460, 2005.

[14] J. Esteban, C. Camacho-Pe nalosa, J. E. Page, T. M. MartinGuerrero, and E. Marquez-Segura, " Simulation of negative permittivity and negative permeability by means of evanescent waveguide modes - theory and experiment," IEEE Transactions on Microwave Theory and Techniques, vol. 53 , no. 4, pp. 1506-1513, 2005.

[15] Y. H. Song, G.-M.Yang and W.Geyi, “ Compact UW bandpass filter with dual notched bands using defected ground structures," IEEE Microwave and Wireless Components Letters, vol. 24, no. 4, pp. 230-232, 2014.

[16] S. Biswas, D. Guha, and C. Kumar, " Control of higher harmonics and their radiations in microstrip antennas using compact defected ground structures," IEEE Transactions on Antennas and Propagation, vol. 61, no. 6, pp. 3349-3353, 2013.

[17] D. Suhas, C. R. Lakshmi, Z. S. Rao, and D. Kannadassan, “ A systematic implementation of elliptic low-pass filters using defected ground structures," Journal of Electromagnetic Waves and Applications, vol. 29, no. 15, pp. 2014-2026, 2015.

[18] Y. L. Zhang, W. Hong, K. Wu, J. X. Chen, and H. J. Tang,“ Novel substrate integrated waveguide cavity filter with defected ground structure," IEEE Transactions on Microwave Theory and Techniques, vol. 53, no. 4, pp. 1280-1286, 2005.

[19] Ahmed Noura, Mohamed Benaissa, Mehadji Abri, Hadjira Badaoui TanHoa Vuong, Junwu Tao, "Miniaturized halfmode SIW bandpass filter design integrating dumbbell DGS cells," microwave and optical technology letters Vol. 61, Iss.6, pp. 1473-1477, June 2019.

[20] Mohamed Amine Rabah, Mehadji Abri, Hadjira Abri Badaoui, Junwu Tao, and Tan-Hoa Vuong, " Compact Miniaturized Half-Mode Waveguide/High Pass-Filter Design Based On Siw Technology Screens Transmit-IEEE C-Band Signals", Vol.58, Iss.2, pp. 414-418, February 2016. 